\title{
Erratum to: ZnO nanorods: morphology control, optical proper- ties, and nanodevice applications [Sci China-Phys Mech Astron, 2013, 56: 2243-2265]
}

\author{
ZHOU WeiYa ${ }^{1}$, ZHANG XiaoXian $^{2}$, ZHAO Duan $^{1}$, GAO Min ${ }^{2} \&$ XIE SiShen ${ }^{1,2^{*}}$ \\ ${ }^{1}$ Beijing National Laboratory for Condensed Matter Physics, Institute of Physics, Chinese Academy of Sciences, Beijing 100190, China; \\ ${ }^{2}$ Key Laboratory for the Physics and Chemistry of Nanodevices and Department of Electronics, Peking University, Beijing 100871, China
}

Received June 13, 2014; accepted June 14, 2014

PACS number(s): 81.07.-b, 81.16.-c, 78.67.-n, 85.60.-q

Citation: Zhou W Y, Zhang X X, Zhao D, et al. Erratum to: ZnO nanorods: morphology control, optical properties, and nanodevice applications [Sci ChinaPhys Mech Astron, 2013, 56: 2243-2265]. Sci China-Phys Mech Astron, 2014, 57: 1816

In the original publication of this paper, the affiliation and superscripted number relating to the current address of the authors has been documented incorrectly. The corrected information appears in this erratum.

\footnotetext{
*Corresponding author (email: ssxie@iphy.ac.cn)
} 